
\section{CCHEST}

Oncology and Transplantation, Department of Medicine, University of Minnesota, Minneapolis, Minnesota, USA ${ }^{2}$ Division of Cardiothoracic Surgery, Department of Surgery, University of Minnesota, Minneapolis, Minnesota, USA

${ }^{3}$ Department of Pathology and Laboratory Medicine, University of Minnesota, Minneapolis, Minnesota, USA

${ }^{4}$ Department of Pulmonary, Critical Care and Sleep Medicine, University of Minnesota, Minneapolis, Minnesota, USA

\section{Correspondence to}

Dr Celalettin Ustun, Division of Hematology, Oncology and

Transplantation, Department of Medicine, University of

Minnesota, 14-100 PWB, 516

Delaware Street SE,

Minneapolis, MN 55455, USA; custun@umn.edu

Received 28 January 2015 Revised 16 March 2015 Accepted 4 April 2015 Published Online First 2 May 2015

\section{CrossMark}

To cite: Ustun C,

Randall N, Podgaetz $\mathrm{E}$, et al.

Thorax 2015:70:701-704.

\title{
Severe dyspnoea in a patient with chronic myelogenous leukaemia on a tyrosine kinase inhibitor
}

\author{
Celalettin Ustun, ${ }^{1}$ Nicole Randall, ${ }_{1}^{1}$ Eitan Podgaetz, ${ }^{2}$ Khalid Amin, ${ }^{3} \mathrm{H}$ Erhan Dincer ${ }^{4}$
}

NR (Haematology fellow): A 78-year-old male patient with chronic myelogenous leukaemia (CML) diagnosed initially in 2011 presented to the haematology clinic with fatigue and dyspnoea at rest and with exertion. His medical history included benign prostatic hyperplasia and a prior diagnosis of acute myelogenous leukaemia (AML) with translocation $8 ; 21$ (good risk AML) diagnosed in 2009, which was in complete remission after standard cytarabine and daunorubicin induction chemotherapy and cytarabine consolidation chemotherapy, requiring no treatment since January 2010. He had also been a smoker, with a 40 pack-year smoking history, who quit 40 years ago. His chest X-ray and CT from 2012 prior to nilotinib therapy demonstrated no focal airspace disease, no evidence of small airways disease and no pleural effusion (figure 1). In July 2013 prior to this current presentation, he had developed a pleural effusion while receiving dasatinib as a second-line agent because of suboptimal response to imatinib. Pleural fluid analysis at that time demonstrated an exudative effusion with $81 \%$ of lymphocyte predominance (395 white blood cells, WBCs) and negative staining and cultures for bacterial, fungal and acid fast bacilli. It was consistent with dasatinib-induced pleural effusion. Dasatinib was switched to nilotinib, and pleural effusion resolved until October 2014 when he presented again with fatigue and dyspnoea to the clinic.

On examination, the patient's heart rate was $82 \mathrm{bpm}$, blood pressure $115 / 70 \mathrm{~mm} \mathrm{Hg}$, respiratory rate 24 breaths/min, $\mathrm{SpO}_{2} 94 \%$ and temperature $97.3^{\circ} \mathrm{F}$. The patient was in mild respiratory distress, actively working to take deep breaths. Respiratory examination was remarkable for poor air entry at the lung bases bilaterally with dullness to percussion to the mid-lung fields posteriorly, right side greater than left. There was no egophony or tactile fremitus. Cardiac examination revealed a regular rhythm with a systolic ejection murmur radiating to the carotid arteries bilaterally.

His WBC count was $7.6 \times 10^{9} / \mathrm{L}$ with a normal differential except for an elevated absolute monocyte count of $2.2 \times 10^{9} / \mathrm{L}$. Haemoglobin was $14.0 \mathrm{~g} / \mathrm{dL}$ and platelet count was $165 \times 10^{9} / \mathrm{L}$. Comprehensive metabolic panel demonstrated normal electrolytes and kidney and liver functions. $B C R / A B L 1$ fusion gene was undetectable by PCR in the peripheral blood (ie, CML was still in molecular remission). Chest CT showed marked diffuse thickening of the pleura in the left hemithorax with a moderate size, slightly loculated pleural effusion (figure 2A).
Echocardiogram revealed normal left ventricular ejection fraction (EF) of 55\% with mild diastolic dysfunction and was otherwise unchanged from baseline echocardiogram in October 2013, which also demonstrated mild diastolic dysfunction, normal left ventricular EF of 55\%-60\%. Baseline ECG in August 2013 demonstrated a normal sinus rhythm, with normal axis and no ST or $\mathrm{T}$ wave abnormalities, and current ECG was unchanged.

In addition, rheumatologic work-up was unremarkable, with negative ANA, p-ANCA, c-ANCA, scleroderma antibody, Smith antibody, SSA/SSB, RNP, Jo-1 antibody and rheumatoid factor testing.

CU (Staff haematologist): CML is a haematopoietic stem cell malignancy with an incidence rate of 1.5 per 100000 individuals per year within the USA. ${ }^{1}$ CML is characterised by the presence of the Philadelphia $(\mathrm{Ph})$ chromosome, which results from a translocation between chromosomes 9 and 22, which results in the pathogenic tyrosine kinase signal transduction protein, BCR/ABL1. The treatment of CML has been dramatically changed by the use of tyrosine kinase inhibitors (TKIs). The first developed was imatinib, which is effective in the majority of patients. Few patients need secondline TKIs (eg, dasatinib or nilotinib) because of resistance or intolerance. ${ }^{2}$ Second-line TKIs are more effective but also have specific adverse effects, including pleural effusion.

In this patient's case, CML was in molecular remission, and the current complete blood counts had no indication of disease progression. His haemoglobin was normal, which could not have been responsible for his dyspnoea.

TKIs have been reported to cause pleural effusions, which are predominantly lymphocytic, and can cause fluid retention. ${ }^{3}$ Lymphocytosis in patients receiving dasatinib has been shown to be associated with pleural effusion and better outcomes. ${ }^{3}$ Although these are rare side effects, dose reduction, interruption of TKI or switching to another TKI may resolve the recurrence of pleural effusion. ${ }^{3}$ Pleural effusions are described with TKI therapy, in particular with dasatinib. ${ }^{4}$ The exact mechanism is not known but postulated to be related to fluid retention from non-specific inhibition of platelet-derived growth factor receptor- $\beta$ or other kinases, ${ }^{5}$ or immune related. ${ }^{6}$ History of cardiac disease, hypertension and use of a twicedaily schedule (vs once daily) were identified as risk factors for the development of pleural effusions. ${ }^{5}$ Perhaps, we are dealing with recurrence of pleural effusion, although this patient was no longer on dasatinib, but rather on nilotinib. Pleural effusions 


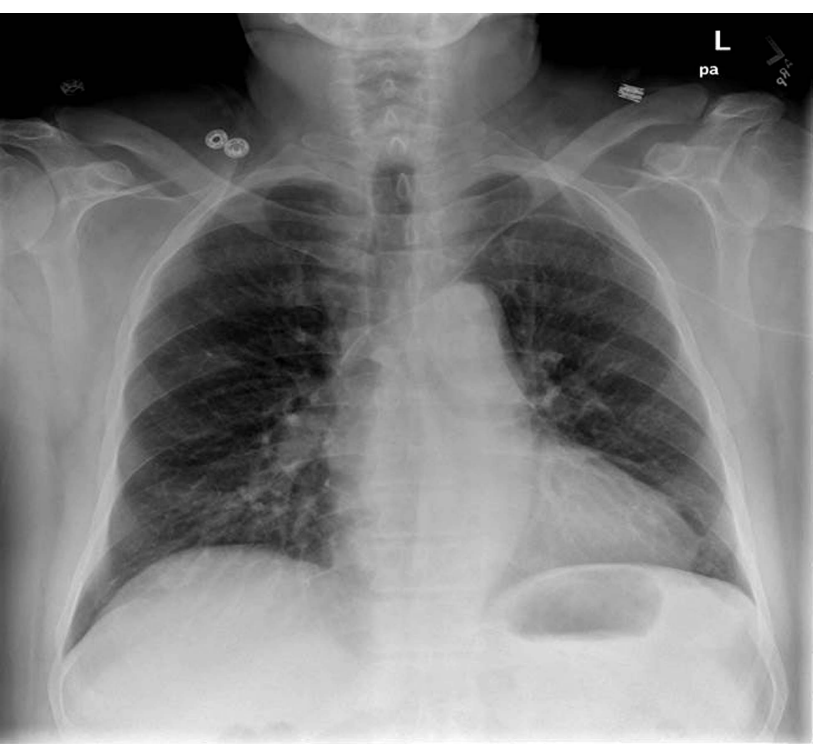

Figure 1 There are no focal airspace opacities, no evidence of pleural effusion or thickening. The cardiac silhouette is not enlarged. The pulmonary vessels are slightly distinct.

are uncommonly associated with niliotinib therapy (1\%). However, at this point there were four important questions: (1) Is nilotinib the sole cause of the patient's pleural effusion? (2) Is the pleural effusion solely responsible for his dyspnoea? (3) What is the reason for the pleural thickening seen on the patient's CT chest imaging, which to our knowledge has not been reported with TKIs? (4) What is the best diagnostic approach to investigate the aetiology of the pulmonary thickening seen on CT chest imaging?

HED (Staff pulmonologist): Although large pleural effusion and diffuse pleural thickening may cause restrictive pulmonary physiology leading to dyspnoea, we also entertained other possibilities in his case such as pulmonary hypertension related to sleep apnoea. However, his echocardiogram was not suggestive of increased pressures in the pulmonary circulation, and there was no evidence of left ventricular systolic dysfunction but mild diastolic dysfunction. Exudative pleural effusion with diffuse pleural thickening has a broad differential diagnosis of chronic infections, such as tuberculosis or fungal infections, and can be seen in partially treated bacterial infections and malignancies (primarily pleural lymphoma or mesothelioma). Diagnostic yield of pleural fluid cultures or cytology is much less than that of pleural biopsy, tissue cultures or pathological evaluation. Therefore, pleuroscopy, a minimally invasive method, allows for examination of pleural surfaces, both parietal and visceral, and the ability to obtain biopsies from the parietal pleura under direct visualisation.

HED and ER (Staff thoracic surgeon): Pleuroscopy with biopsies and pleural fluid drainage was performed for diagnostic purposes. Intraoperatively, we found diffusely thickened parietal pleura with multiple adhesions and fibrotic changes creating innumerable pockets of loculations. Parietal pleural biopsies showed organising fibrinous pleuritis (OFP) with no evidence of abnormal cellular infiltrate, granuloma or malignancy (figure 3 ). Tissue and fluid cultures from the pleura and pleural effusion were negative for bacteria, fungi, actinomyces and acid fast bacilli. The effusion was predominantly composed of lymphocytes. Flow cytometry of pleural fluid was normal and demonstrated polytypic B cells, with no aberrant immunophenotype on $\mathrm{T}$ cells and rare to absent blasts.
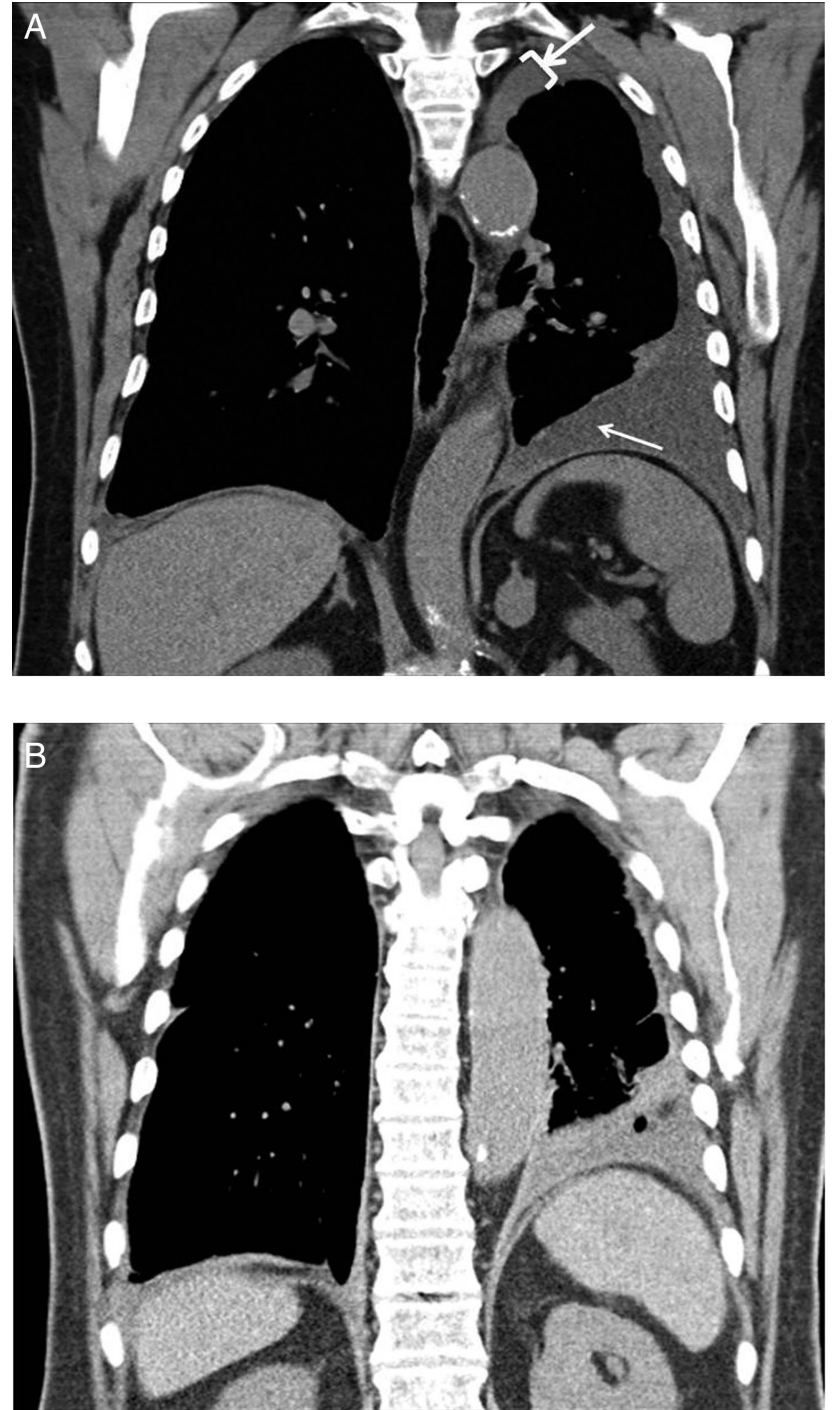

Figure 2 (A) Marked diffuse pleural thickening (wide arrow) on the entire left side enclosing lung and loculated pleural effusion (narrow arrow). (B) Improved pleural effusion but no change in pleural thickening in 2 months in the follow-up CT.

HED and KA (Staff pathologist): OFP can coexist with various malignant or infectious conditions in the pleural space. Pleuritis is a reactive process which may include inflammatory cell infiltrates. There may be fibrinous exudates on the pleural surface (fibrinous pleuritis), organisation of the fibrin by granulation tissue (organising pleuritis) or maturing connective tissue (fibrous pleuritis). OFP is an acellular fibrous thickening of the pleura with focal plaque-like features. OFP can be associated with various disorders, including cardiovascular disease, tuberculosis, mesothelioma, lymphoma and metastasis. ${ }^{8}$

$C U$ : All the causes of OFP were excluded, and therefore the most likely possible explanation as a cause of pleural thickening was TKI; perhaps, the patient's OFP started with dasatinib use and worsened with nilotinib use with time. This may represent one of the first cases of this phenomenon; however, it is not possible to definitely identify this finding as this is a diagnosis of exclusion. To our knowledge, this is the first case reported in the English literature. Interestingly, there appears to be no evidence that TKIs, such as nilotinib, contribute to fibrosis in the heart or lung; rather, they may exert antifibrotic effects, as seen in liver and lung animal models. ${ }^{9-11}$ The possible antifibrinotic 

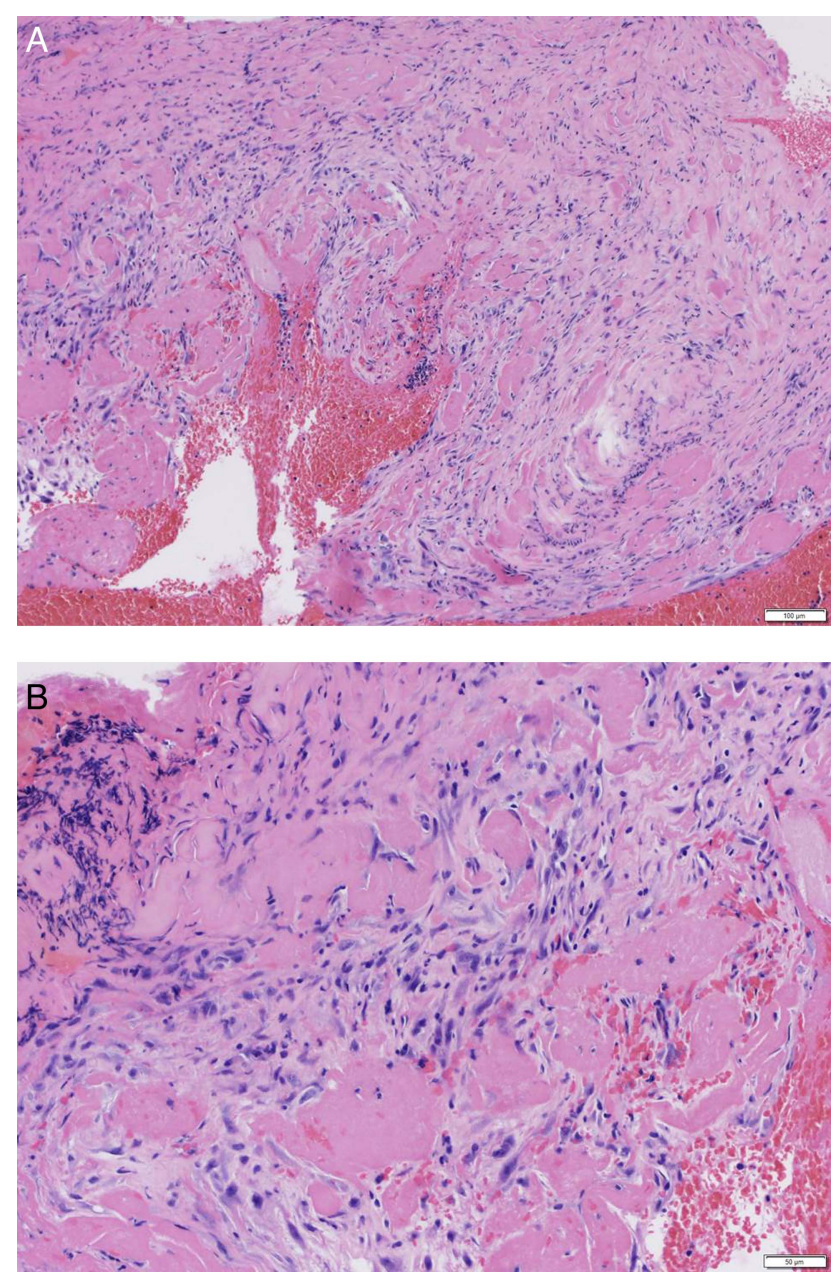

Figure 3 Fibrin deposits with granulation tissue formation, acute and chronic inflammation and early fibrosis.

mechanism of action of TKIs is in part due to lowering transforming growth factor levels decreasing the platelet-derived growth factor. $^{9-11}$

Methylprednisolone $40 \mathrm{mg}$ daily by mouth was given for 4 weeks with taper, and nilotinib was replaced by low-dose omacetaxine, a non-TKI drug $\left(1.25 \mathrm{mg} / \mathrm{m}^{2}\right.$ twice a day for 3 days) with clinical improvement in the patient's breathing and fatigue.

NR: He was admitted a few days later. He was pancytopenic (WBC count had decreased to $0.7 \times 10^{9} / \mathrm{L}$ with an absolute neutrophil count of $0.0 \times 10^{9} / \mathrm{L}$, haemoglobin of $8.5 \mathrm{~g} / \mathrm{dL}$ and platelet count of $23 \times 10^{9} / \mathrm{L}$ ) due to omacetaxine, and his $\mathrm{N}$-terminal pro-brain natriuretic peptide was found elevated above the baseline to $547 \mathrm{pg} / \mathrm{mL}$ (reference lab states that $0-450 \mathrm{ng} / \mathrm{mL}$ rules out congestive heart failure in an outpatient aged 75 years or older). Echocardiogram showed mild diastolic dysfunction, and normal global and regional left ventricular function with an EF of $60 \%-65 \%$, with no significant valvular abnormalities. One possible explanation was that his diastolic dysfunction could be age related; it is unlikely to be due to chronic hypertension, diabetes mellitus or coronary artery disease, as he did not have these underlying conditions. $\mathrm{He}$ also had moderate pleural effusion, and thoracentesis was performed. Microbiology, chemistry and cell count analysis of the pleural effusion were all similar to that of the patient's prior effusion levels.
He was discharged to a rehabilitation centre and was followed up frequently. A month later, his pancytopenia improved; he was off steroids and nasal oxygen support. However, he still had exertional dyspnoea. Chest CT and pulmonary function testing (PFT) were repeated.

HED: His chest CT scan showed minimal pleural effusion, no airspace disease and unchanged pleural thickening (figure 2B). PFTs showed a restrictive pattern (total lung capacity (TLC) $52 \%$ of predicted), which was much worse than a year ago when TLC was $72 \%$ of predicted. The pleural thickening seen on chest CT is likely responsible for the restrictive process seen on PFTs and explains his improved, but ongoing dyspnoea.

$C U$ : Although his respiratory symptoms and pleural effusion improved, pleural thickening persisted. It is unknown whether fibrous pleuritis is irreversible at this point of time, but clearly it has not improved in the 2 months since nilotinib was discontinued. Moreover, he developed severe pancytopenia on the alternative drug, omacetaxine. This case demonstrates that pleural complications of TKI can be severe and hinder treatment of CML. Almost half the number of patients with CML on secondgeneration TKIs experience side effects, including pleural effusion in $26 \%$ of patients on dasatinib and $2 \%$ on nilotinib. ${ }^{12}$ Side effects are often a reason for the difficulty patients have with adhering to these lifelong drugs. ${ }^{12}$ Unfortunately, although there are multiple TKIs, there can be cross reactions between them regarding adverse effects. Other non-TKI options are limited and have different but severe toxicities, as demonstrated in our patient. Allogeneic haematopoietic cell transplantation can be considered in younger patients who cannot tolerate TKIs; however, this is not a good option for this 78-year-old patient. ${ }^{13}$ However, long-term outcomes in patients who are able to tolerate TKI therapy are good with an overall 5 -year survival of $93 \%$, but this decreases in older adults (defined as age $30-85$ years) to $82 \% .^{14}$

Contributors All contributing authors were involved in the care of the patient. CU planned and prepared the first draft of the manuscript. All authors contributed to the development of the final version of the manuscript. CU is responsible for the overall content of the manuscript as guarantor.

Competing interests None declared.

\section{Patient consent Obtained.}

Provenance and peer review Not commissioned; externally peer reviewed.

\section{REFERENCES}

1 Bartram CR, de Klein A, Hagemeijer A, et al. Translocation of c-ab I oncogene correlates with the presence of a Philadelphia chromosome in chronic myelogenous leukaemia. Nature 1983;306:277-80.

2 Kantarjian HM, Talpaz M, Giles F, et al. New insights into the pathophysiology of chronic myeloid leukemia and imatinib resistance. Ann Intern Med 2006;145:913-23.

3 Eskazan AE, Eyice D, Kurt EA, et al. Chronic myeloid leukemia patients who develop grade I/II pleural effusion under second-line dasatinib have better responses and outcomes than patients without pleural effusion. Leuk Res 2014;38:781-7.

4 Masiello D, Gorospe G, Yang A. The occurrence and management of fluid retention associated with TKI therapy in CML, with a focus on dasatinib. $J$ Hematol Oncol 2009;2:46.

5 Quintas-Cardama A, Kantarjian H, O-Brien S, et al. Pleural effusion in patients with chronic myelogenous leukemia treated with dasatinib after imatinib failure. J Clin Oncol 2007;25:3908-14.

6 De Lavallade H, Punnialingam S, Milojkovic D, et al. Pleural effusions in patients with chronic myeloid leukaemia treated with dasatinib may have an immune-mediated pathogenesis. Br J Haematol 2008;141:745-7.

7 Kantarjian HM, Giles F, Gattermann N, et al. Nilotinib (formerly AMN107), a highly selective $B C R-A B L$ tyrosine kinase inhibitor, is effective in patients with philadelphia chromosome-positive chronic myelogenous leukemia in chronic phase following imatinib resistance and intolerance. Blood 2007;110:3540-6.

8 Metintas M, Ak G, Cadirci 0, et al. Outcome of patients diagnosed with fibrinous pleuritis after medical thorascopy. Respir Med 2012;106:1177-83. 


\section{Chest clinic}

9 Distler JHW, Distler O. Intracellular tyrosine kinases as novel targets for antifibrotic therapy in systemic sclerosis. Rheumatology 2008;47:V10-11.

10 Shiha GE, Abu-Elsaad NM, Zalata KR, et al. Tracking anti-fibrotic pathways of nilotinib and imatinib in experimentally induced liver fibrosis: an insight. Clin Exper Pharm Physiol 2014;41:788-97.

11 Shaker ME, Shiha GE, Ibrahim TM. Comparison of early treatment with low dosesof nilotinib, imatinib, and a clinically relevant dose of silymarin in thrioacetamide-induced liver fibrosis. Europ J Pharm 2011;670:593-600.

12 Gora-Tybor J, Medras E, Calbecka M, et al. Real-life comparison of severe vascular events and other non-hematological complications in patients with chronic myeloid leukemia undergoing second-line nilotinib or dasatinib treatment. Leuk Lymphoma Published Online First: 5 Mar 2015. doi:10.3109/ 10428194.2014.994205

13 Pemmaraju N, Cortes J. Chronic myeloid leukemia in adolescents and young adults: patient characteristics, outcomes and review of the literature. Acta Haematol 2014;132:298-306.

14 Pemmaraju N, Kantarjian H, Shan J, et al. Analysis of outcomes in adolescents and young adults with chronic myelogenous leukemia treated with upfront tyrosine kinase inhibitor therapy. Haematologica 2012;97:1029-35. 